\title{
New Developments in Breast Cancer Therapy Presented at ASCO 2010
}

\author{
Chair: Volkmar Müller ${ }^{\mathrm{a}}$ \\ Participants: Peter Dubsky ${ }^{\mathrm{b}}$ Tanja Fehm ${ }^{\mathrm{c}}$ Florian Fitzal $^{\mathrm{d}}$ Jens Huober \\ Marcus Schmidt ${ }^{f}$ Günther Steger ${ }^{g}$

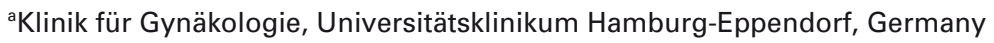

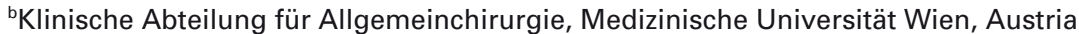 \\ 'Universitätsfrauenklinik Tübingen, Germany \\ ${ }^{\mathrm{d} A b t e i l u n g}$ für Allgemeinchirurgie, Brustgesundheitszentrum, Medizinische Universität Wien, Austria \\ 'Brustzentrum, Kantonsspital St. Gallen, Switzerland \\ ${ }^{\mathrm{f} K l i n i k}$ und Poliklinik für Frauenheilkunde und Geburtshilfe, Klinikum der Johannes Gutenberg-Universität, Mainz, Germany \\ ${ }^{9}$ Klinische Abteilung für Onkologie, Universitätsklinik für Innere Medizin I, Medizinische Universität Wien, Austria
}

\section{Question 1: Which New Developments in Targeted Therapies of Breast Cancer Patients Were Presented at the Meeting that Will Find Their Way into Your Practice?}

Dubsky: Endocrine treatment: One of the highlights was the contribution by the Austrian Breast and Colorectal Cancer Study Group (ABCSG) concerning the impact of BMI on endocrine treatment (Disclosure: I am a co-author). The study investigated the impact of BMI in premenopausal patients undergoing endocrine treatment with goserelin combined either with tamoxifen or anastrozole (retrospective analysis of patients from ABCSG-12). Overweight patients treated with anastrozole double their risk of death. No such impact of BMI is seen in tamoxifen treated patients. It is noteworthy that these results were confirmed in a retrospective analysis of the ATAC trial published shortly after the ASCO meeting (Sestak et al.; J Clin Oncol 2010;28:3411-5.).

M. Ellis (LBA513) presented data from ACOSOG Z1031, a randomized phase II trial using exemestane, anastrozole or letrozole in neoadjuvant endocrine therapy. 374 women with large, estrogen receptor (ER)-rich tumors were treated for 16 weeks with one of the aromatase inhibitors (AI) and included in the ITT analysis. This study demonstrates very little difference between the 3 AI candidates. The clinical remissions shown and the breast conservation rates in these large tumors (median tumor size $4.0 \mathrm{~cm}$ ) together with low disease progression rates add to the growing body of evidence supporting both safety and efficacy of preoperative endocrine therapy.
ACOSOG has recently amended the protocol and refined their methodology. Patients will receive biopsies 2 weeks after starting therapy. KI67 decrease (or the lack thereof) will be assessed and patients with a lack of endocrine response chance will be selected earlier for cytotoxic treatment.

Anti-HER2 treatment: Again, there were no data with immediate practice changing potential. However, there are two fairly new agents that show potent anti-tumor activity in a number of reports. Pertuzumab is a monoclonal humanized antibody targeting the extracellular domain with a binding site distinct from trastuzumab. Importantly, it seems to inhibit heterodimerization of HER2 and HER3 in a highly effective manner. The compound has shown considerable activity in pretreated patients, and will be further developed in combination with trastuzumab upon earlier trastuzumab failure. Abstract 1066 showed an interesting biomarker study that suggests low expression of mRNA of HER2 and HER3 to have prognostic and predictive potential under pertuzumab/ trastuzumab treatment.

T-DM1 is a conjugate of trastuzumab and a cytotoxic agent. As with pertuzumab, early data show efficacy of this compound in patients pretreated with trastuzumab and/or lapatinib. It is currently being compared to standard treatment options in phase III clinical trials. Abstracts 1012 (K. Miller) and 1016 show substantial efficacy with encouraging toxicity data of T-DM1. Interestingly, biomarker analysis show initial data that would suggest less activity in patients with decreased PTEN or PI3K mutations.

\section{KARGER}

Fax +497614520714

Information@Karger.de

www.karger.com (c) 2010 S. Karger GmbH, Freiburg

Accessible online at:

www.karger.com/brc
PD Dr. Volkmar Müller

Klinik und Poliklinik für Gynäkologie

Universitätsklinikum Hamburg-Eppendorf

Martinistrasse 52, 20246 Hamburg, Germany

Tel. +4940 7410-57606, Fax -40070

v.mueller@uke.de 
Anti-VEGF treatment: Joyce O'Shaughnessy reported on a metaanalysis of Ribbon-1, E2100 and AVADO. Avastin produces clear-cut improvement of progression free survival. There is no indication of a clinically meaningful overall survival effect. This surgical oncologist was disappointed to find very little news on anti-EGFR or anti-IGF treatments.

Fehm: Resistance to trastuzumab may be associated with deregulation of the PI3K pathway. Inhibition of mTOR via everolimus seems to overcome trastuzumab-resistance. Two phase II trials for trastuzumab resistant HER2 overexpressing breast cancer were presented at this ASCO meeting, combining everolimus with paclitaxel or trastuzumab, respectively. The combination was well tolerated and showed promising clinical activity. Although the data have to be verified in larger phase III studies, everolimus has a great potential for the treatment trastuzumab resistant HER2-overexpressing breast cancer.

Huober: Several phase I/II trials showed promising activity of new agents like pertuzumab, RAD 001 or T-DM1 in patients with trastuzumab failure and HER2-positive tumors. Further first results of PI3K inhibitors showed that these drugs have clinical activity.

Schmidt: PARP inhibitor trials presented at this years ASCO were promising. In terms of approved therapies bevacizumab is still a major topic especially for patients with an urgent need for remission. Sunitinib failed to show efficacy in two trials in advanced breast cancer and has thus no role in this disease setting.

Steger: T-DM1 and pertuzumab seem to be promising.

\section{Question 2: Which Results with Relevance for the Treat- ment of Triple Negative Breast Cancer Did You Find that Should Lead to Progress in the Clinical Management?}

Dubsky: The role of platinum based compounds in breast cancer remains unclear. Further development in triple negative breast cancer (TNBC) is ongoing. Cetuximab, an anti-EGFR agent has shown promise in TNBC and will be further developed. There were no new data of BSI-201 with relevance to clinical management. Olaparib an oral PARP inhibitor showed a favorable toxicity profile in combination with e.g. weekly paclitaxel (abstract 1018). There is increasing evidence suggesting that oral PARP inhibitors administered without a chemotherapy backbone seem to have an anti-tumor activity limited to mutation carriers of BRCA (abstract 3002).

Fehm: Promising therapeutic strategies have been demonstrated based on EGFR (e.g. cetuximab) or PARP (e.g. olaparib, BSI-201) inhibition. Antiangiogenesis may also contribute to improved outcome in these patients. The role of platinum based compounds in TNBC is still under investigation. Until solid data are available, no specific treatment strategies can be recommended for this subgroup of patients.

Huober: Not all triple negative tumors are the same and it will be the challenge for the future to identify those which will likely respond to PARP inhibitors.

Schmidt: Certainly PARP inhibitors are a major topic in TNBC. Encouraging were promising results for docetaxel in primary breast cancer showing that basal-like breast cancer, which is mostly triple negative, is especially sensitive to taxanes. These results contrast earlier findings claiming a resistance against taxanes in triple negative / basal-like breast cancer.

Steger: None with a level of evidence sufficient for daily routine but PARP-inhibitors still look very interesting.

\section{Question 3: Are Bisphosphonates as Anticancer Treatment in Early Breast Cancer Part of Your Clinical Practice? What Are Your Reasons for this Decision?}

Dubsky: I was an investigator in ABCSG-12, the first trial to suggest anti tumor efficacy of zoledronic acid (ZA) in the adjuvant setting. The initial results from ABCSG-12 have matured very well and the updated results confirm a one third reduction in risk of recurrence. It is important to stress that the updated subgroup analysis suggests no interaction with the type of endocrine treatment used in the trial. Furthermore, it will be interesting to see if the trend in improved overall survival will become a significant finding. I am currently using ZA in my daily practice but the use is strictly limited to patients with inclusion criteria of ABCSG-12: this means premenopausal patients with endocrine responsive breast cancer scheduled for combined endocrine treatment with goserelin and tamoxifen in the absence of adjuvant chemotherapy (but not neoadjuvant chemotherapy).

Fehm: Based on the results of the ABCSG-12 and the ZFAST/ZoFAST trial we offer bisphosphonates to premenopausal breast cancer patients with GnRH and tamoxifen and to postmenopausal patients with AI.

Fitzal: Bisphosphonates have been included into my clinical practice since our trial ABCSG-12 and after the results I prescribe the bisphosphonate ZA for premenopausal women adjuvant with hormone receptor-positive breast cancer for 3 years. This has not changed after the meeting, however, denosumab seems to be a highly active drug which may replace $\mathrm{ZA}$ in future as seen in a pancreatic cancer study with metastasized patients and a clear reduction of skeletal events by denosumab application compared with ZA. 
Huober: The routine use of bisphosphonates for all women with normal bone health receiving adjuvant endocrine therapy can not be considered standard. Reasons for this are the low number of events, the fact that most benefit in the ABCSG-12 trial was seen with a treatment ( GnRH and anastrozole) which is not considered standard, and lack of overall survival benefit so far. However, bisphosphonates are obviously a reasonable option for individual patients. Several trials will elucidate the role of bisphosphonates in the adjuvant setting in the near future and may support the wide use of those agents in the adjuvant setting.

Schmidt: At present I prescribe ZA in early breast cancer only to premenopausal patients with $\mathrm{GnRH}+$ tamoxifen treatment due to the encouraging results of the ABCSG-12 trial.

Steger: Yes, based on the results of ABCSG-12 we offer bisphosphonates to premenopausal patients with hormone-sensitive tumors in the adjuvant setting.

\section{Question 4: Which of the Presented Data on Adjuvant and Neoadjuvant Chemotherapy Will Be Applied in Your Clinical Routine?}

Dubsky: Concerning neoadjuvant chemotherapy the ABCSG has previously shown the efficacy of concomitant epirubicin and docetaxel $(\mathrm{E}+\mathrm{T})$. Recently, the integration of capecitabine $(\mathrm{X})$ into the regimen has been shown to improve pathologic complete remission ( $\mathrm{pCR}$ ) rates with acceptable additional toxicity. Abstract 530 confirms previous reports of neoadjuvant chemotherapy suggesting that patients with hormone receptor (HR)-negative disease and poor differentiation have greater benefit from preoperative cytotoxic treatment. In my own practice I will propose the $\mathrm{E}+\mathrm{T}$ with $\mathrm{X}$ regimen to young (and fit) patients with several features of breast cancer that suggest little or no response to endocrine treatment. There were no reports concerning adjuvant chemotherapy that will change our current regimens of adjuvant chemotherapy.

Fehm: Based on the data of the Adebar trial obese patients respond less to adjuvant chemotherapy indicating that lifestyle and nutrition counseling should be part of the follow-up of breast cancer patients. Regarding neoadjuvant chemotherapy, von Minckwitz et al. presented a pooled multilayer analysis of 8 German neoadjuvant chemotherapy trials. Based on the results obtained from more than 3,300 patients HR-positive tumors benefit more from longer neoadjuvant treatments, HER2-negative tumors more from higher anthracycline doses, triple negative tumors from short-term higher dose taxane and anthracycline based treatment, and all subtypes from the addition of capecitabine. Therefore, the subtype of the primary tumor will become more important for designing neoadjuvant clinical trials.

Fitzal: Capecitabine seems to be highly active in neoadjuvant chemotherapy for breast cancer within all risk subgroups. Anastrozol may not yield such an increased improvement for HR-positive breast cancer patients and is still not to be used for premenopausal patients. OncotypeDX may also act as predictive marker for chemotherapy decision in HR-positive breast cancer patients. Disseminated tumor cells in the bone marrow do not seem to play an important role for the prognosis of node-negative breast cancer patients.

Huober: At this year's ASCO the trials about locoregional treatment will likely cause a change in clinical practice. In a selected population it does not seem to be necessary to do further axillary dissection even if the sentinel node is positive; and in elderly HR-positive patients with pT1 tumors radiotherapy to the breast was of little additional effect in the presence of tamoxifen treatment. Those data suggest that in a selected population less local treatment in the presence of adjuvant systemic therapy does not result in worse local and systemic control.

Schmidt: There were only sparse data on adjuvant or neoadjuvant chemotherapy. Most compelling were the results of the German neoadjuvant trials showing that especially ER-positive patients profit from longer neoadjuvant chemotherapy.

\section{Question 5: Genomic Signature and Early Breast Cancer. Which New Aspects Were Presented that Will Influence Your Patient Management?}

Dubsky: We are currently not using any of the commercially available tests in routine patient management. Indeed, prospective data testing genomic signatures as biomarkers are still lacking. We have recently initiated a study that will help us to evaluate the implementation of genomic signatures in our decision-making process. I think it is vital that we specify which patients are most likely to receive additional prognostic and predictive information from genomic signatures. M. Knauer presented new data concerning the Mammaprint signature in relation to histologic grading (abstract 561). It is interesting that the signature is able to identify a subgroup of G1 tumors with poor outcome. This finding adds to the general notion that ER-positive breast cancers must be seen as a heterogeneous group despite several pathologic features suggestive of good outcome.

Fehm: Based on the results obtained by retrospective studies, gene expression profiles may have a great potential to opti- 
mize treatment decisions particularly in breast cancer patients with intermediate risk. However, to implement these new tools routinely in the patient's management, the clinical value has to be proven in prospective clinical trials. These trials including e.g. MINDACT, Plan B, and TAILORx are all still recruiting and clinical data will not be available in the near future.

Fitzal: OncotypeDX may have a predictive value. It may be included in standard breast cancer diagnostics to decide whether a patient needs chemotherapy in endocrine responsive disease or not. However, these data need validation with a sophisticated trial for this gene expression profile as predictive marker.

Huober: The evaluation of gene signatures is still a matter of research and should be further investigated. There were no new data about gene signatures at the ASCO meeting which will now influence patient management. There are discussions if a quality controlled immunohistochemistry will be as good as gene signatures in clinical practice. Moreover, the integration of clinical parameters in addition to the results of gene arrays seems to be of importance.

Schmidt: From a practical point of view, incorporation of clinical features like tumor size or nodal status is promising and will find a way into clinical practice. From a scientific standpoint, results showing the independent importance of constituents of the humoral immune system either alone or in conjunction with an Il-8 metagene are interesting, potentially leading to better prognostic assessment especially of triple negative / basal-like breast cancer.

\section{Question 6: Aromatase Inhibitors in the Adjuvant Setting - Upfront or not Upfront? What Are the Considerations that Influence Your Decision?}

Dubsky: In my own clinical practice upfront use of AI is the most common regimen. Although upfront AI have not been shown to decidedly decrease the number of breast cancer deaths, the general toxicity profile is preferable to tamoxifen and the improvement in recurrence rates is sustained and fairly homogenous in all subgroups. The fact that the differences in outcome from upfront and sequencing strategies are really minute should be seen as an opportunity to individualize therapy in case of side effects. Outside of clinical trials compliance with anti-hormonal therapy is most likely an underrated factor.

Fehm: In general, we prefer the 'upfront' strategy since the risk for recurrence is highest in the first 2 years. If the patient has major side effects which are not manageable, we switch to tamoxifen. In case the patient is perimenopausal or has just become postmenopausal, we start with tamoxifen and may switch after 2 or 3 years to an AI if the patient has become definitely postmenopausal. In low-risk situations tamoxifen is still an important option.

Fitzal: This question has not been resolved at the ASCO and there were only few papers regarding this issue. In fact, anastrozole is still not indicated in premenopausal women, ABCSG-12 showed a reduction in overall survival for anastrozole treated patients. In postmenopausal women ABCSG-8 showed only marginal improvement with anastrozole. Compliance seems to be similar between the two groups as well as cognitive function. There may be a beneficial effect regarding overall survival for tamoxifen in overweight patients, however, this has only been shown in premenopausal but not for postmenopausal women and in fact, overweight patients had no differences in disease free survival comparing anastrozole and tamoxifen, thus the difference may be due to side effects of anastrozole in overweight patients rather than in improved breast cancer outcome. Moreover, in normal weight patients there was no difference between tamoxifen and anastrozole, thus the BMI may not be used as predictive marker to decide between these two drugs.

Huober: In postmenopausal HR-positive patients with an intermediate or high risk profile due to the increased relapse during the first 2 years I prefer the upfront approach. In low-risk patients tamoxifen is still an option. However, differences between the treatment strategies may be small and tolerability of the treatment, influence on general health status and obviously patient's wish are important factors for decision-making.

Schmidt: From my point of view, there are only few reasons to give AI upfront, unless there is a contraindication against tamoxifen. In my personal practice I prefer the switch strategy for postmenopausal patients. The only reason for upfront therapy, except for tamoxifen contraindication, is a very high risk for early metastasis (e.g. >9 lymph nodes involved).

Steger: We mostly use the upfront strategy, only in low risk tumors characterized e.g. by low grade and low proliferation rate sequencing of tamoxifen and an AI may be considered.

\section{Question 7: As New Activity at the ASCO Annual Meeting this Year, a 'Trials in Progress' Session Was Established. What Are in Your Opinion Important Breast Cancer Trials Currently Recruiting?}

Dubsky: I did not have the chance to visit this new activity due to conflicting schedules. Later, I have been able to very quickly get an overview of trials in progress using the ClinicalTrials.gov homepage. I am not sure what the advantage of 
such an activity could be in a meeting that is already notoriously busy for most oncologists.

Fehm: I did not attend the session. However, particulary the German study groups have presented their clinical trials including e.g. SENTINA, ICE II, SOFIA, Plan B and the VITAL study.

Fitzal: I did not attend this session.

Huober: How to do axillary staging when neoadjuvant chemotherapy is planned is still an important open question. Thus the German SENTINA trial and a phase II trial of the American college of surgeons also evaluating the sentinel node procedure after neoadjuvant chemotherapy are interesting and important trials. An Italian trial investigating the combination of chemotherapy and metformin in patients with metastatic breast cancer may also show intriguing results.

Schmidt: Several highly interesting studies were presented at the trials in progress poster session. Arguably the most relevant for primary breast cancer in Germany presented here is the WSG Plan B trial comparing an anthracycline-containing chemotherapy regimen (EC 4 - Doc 4), with an anthracycline-free taxane-based regimen (TC 6) in patients with HER2-negative BC. In order to assess whether molecularbased risk assessment may prevent overtreatment, Plan B incorporates the 21-gene recurrence score (RS) as mandatory risk stratification in HR-positive patients with 0-3 involved lymph nodes.

Steger: Out of the plethora of ongoing trials in this field, MINDACT and TAILORx are important to elucidate the role of genomic signatures for treatment selection. ABCSG-25/PANTHER and ABCSG-30/BETH are important to give information about tailoring chemotherapy schedules according to individual patient parameters, and to study bevacizumab in the adjuvant setting, respectively. ABCSG-18 will give data about the possibility to prevent/mitigate clinically relevant treatment induced side effects from AI with denosumab.

\section{Question 8: Which Further Aspects Did You Find of Relevance?}

Dubsky: CRA1004: Women with advanced breast cancer given the experimental chemotherapy drug, eribulin mesylate in a large phase III trial lived longer than those treated with standard cancer therapies - women taking eribulin lived an average of 13.1 months compared to 10.7 months for those receiving conventional cancer treatment. Certainly I would like to see these data go through a peer review process, but they suggest a potent anti-tumor activity in late stages of disease and lines of therapy.
Furthermore, the meeting gave ample opportunity to discuss the issue of biopsy in metastatic cancer. G. Curigliano (CRA1008) reported on 255 consecutive patients with matched primary and liver tissue samples assessed for ER, progesterone receptor (PR), and HER2 status. The receptor status in the liver metastasis differed from the primary in a magnitude that changed targeted treatment in $12.1 \%$ of patients. Conclusions from this and other previous studies should be drawn with care. Indeed, there is little evidence to suggest that the change in treatment leads to prolonged survival. However, visceral metastases occurring late after adjuvant treatment are likely to show a change in receptor status. At the same time interventional radiology performing ultrasound or CT guided biopsies is associated with very low morbidity and - in relation to the targeted therapies - with low cost.

Fehm: Predictive markers including ER, PR and HER2 may change during disease progression. Nevertheless, re-biopsy of the metastatic leasion is not routinely performed. Three abstracts demonstrated that changes of ER, PR and HER2 can occur and therefore may alter therapy strategies in 15$20 \%$ of our patients. Therefore, oncologists and their patients should be motivated to consider a biopsy of the metastatic lesion to avoid an ineffective treatment.

Fitzal: Giuliano presented the data of an early closed trial for N1 patients of the sentinel node with or without axillary dissection ACOSOG Z0011. 25\% of patients in the axillary dissection group had another positive node. There was no increased local or distant recurrence, no difference in OS in patients with or without axillary dissection. It is of notice that the trial was closed early and did not reach the number of patients needed. NSABP B32 showed updated results comparing sentinel node versus axilla in node-negative patients and there was no difference within the two groups in any oncologic parameter demonstrating that the sentinel node biopsy is clearly oncologically safe.

Huober: An investigation from the Dutch cohort of the TEAM trial could not confirm CYP2D6 as an predictor of efficacy of tamoxifen. However, they found that polymorphism of the ERS1 (estrogen receptor 1) impacted disease free survival in breast cancer patients treated with adjuvant tamoxifen. Since there are conflicting data CYP2D6 is not ready for general use in clinical practice.

Schmidt: From a surgical perspective, the ACOSOG Z0011 trial showing no trend toward clinical benefit of axillary lymph node dissection for patients with limited nodal disease is provocative and compelling albeit not yet practice changing. Another important result is that for elderly women with ER-positive, node-negative breast cancer lumpectomy with tamoxifen therapy, but without the addition of radiation, is an 
appropriate treatment option. These two trials are a further step towards reducing loco-regional treatment and thus reducing side effects in selected patients with early breast cancer.

\section{Teilnehmer:}

PD Dr. med. Peter Dubsky

Klinische Abteilung für Allgemeinchirurgie

Medizinische Universität Wien

Währinger Gürtel 18-20, 1090 Wien, Austria

Tel. +43 1 40400-6916, Fax -6918

peter.dubsky@meduniwien.ac.at

Prof. Dr. Tanja Fehm

Universitätsfrauenklinik Tübingen

Calwer Str. 7, 72076 Tübingen, Germany

Tel. +49 7071 29-82211, Fax -4663

tanja.fehm@med.uni-tuebingen.de

a.o. Univ.-Prof. Dr. Florian Fitzal

Abteilung für Allgemeinchirurgie

Brustgesundheitszentrum

Medizinische Universität Wien

Währinger Gürtel 18-20, 1090 Wien, Austria

Tel. +431 40400-5621, Fax -5641

florian.fitzal@meduniwien.ac.at

Prof. Dr. med. Jens Huober

Brustzentrum

Kantonsspital St. Gallen

9007 St. Gallen, Switzerland

Tel: +41 71 494-1177, Fax -6368

jens.huober@kssg.ch

Dr. Marcus Schmidt

Klinik und Poliklinik für Frauenheilkunde und Geburtshilfe

Klinikum der Johannes Gutenberg-Universität

Langenbeckstrasse 1, 55101 Mainz, Germany

Tel. +49 6131 17-2683, Fax -6644

marcus.schmidt@unimedizin-mainz.de

Univ.-Prof. Dr. Güther Steger

Klinische Abteilung für Onkologie

Universitätsklinik für Innere Medizin I

Medizinische Universität Wien

Währinger Gürtel 18-20, 1090 Wien, Austria

Tel. +431 40400-5459 Fax -6081

guenther.steger@meduniwien.ac.at 